\title{
Simultaneous Renal Transplantation and Native Nephrectomy in Patients With Autosomal-Dominant Polycystic Kidney Disease
}

\author{
P. Nunes, A. Mota, R. Alves, A. Figueiredo, B. Parada, F. Macário, and F. Rolo
}

\begin{abstract}
Our objective was to study the influence on transplant outcome of unilateral native nephrectomy of massively enlarged kidneys at the time of renal transplantation among patients with end-stage renal disease owing to autosomal-dominant polycystic kidney disease (ADPKD).

Patients and Methods. We studied 159 renal transplants in patients with ADPKD divided into two groups according to the need to perform a unilateral native nephrectomy owing to enlarged kidneys $(\mathrm{N}+; n=143)$ versus those not $(\mathrm{N} 0 ; n=16)$ needing this procedure. Parameters related to the donors, grafts, recipients, and operative data were correlated with short- and long-term outcomes. The groups were homogeneous in terms of recipient and donor ages, genders, HLA compatibilities, and length of pretransplant dialysis.

Results. When no nephrectomy was needed surgery length was shorter (N0, $3.01 \mathrm{vs.} \mathrm{N}+$, 4.23 hours; $P<.001)$, less intraoperative crystalloids were infused (N0, 1.84 vs. $\mathrm{N}+, 2.76$ $\mathrm{L} ; P<.001)$, and less plasma (N0, 2.07 vs. $\mathrm{N}+, 2.93 \mathrm{U} ; P<.05)$, or blood (N0, 1.05 vs. $\mathrm{N}+$, $1.81 \mathrm{U} ; P<.05)$ transfusions were required. Hospital stay was similar $(\mathrm{N} 0,12.70 \mathrm{vs} \mathrm{N}+$, 16.50 days; $P$ not significant $[\mathrm{NS}])$. There was only one urologic complication in the nephrectomy group. There were no differences $(P=\mathrm{NS})$ in rates of delayed graft function $(\mathrm{N} 0,19.9 \% ; \mathrm{N}+, 12.5 \%)$, acute rejections $(\mathrm{N} 0,25.5 \% ; \mathrm{N} 0,33.3 \%)$, chronic allograft dysfunction (N0,15.8\%; $\mathrm{N}+, 28.6 \%$ ). Graft function at 1 month as well as 1 and 5 years were comparable. Patient and graft survivals were similar at 1 and 5 years. There were no differences in the causes of graft loss or patient death.

Conclusion. In patients with ADPKD native nephrectomy of massively enlarged kidneys may be safely performed during the transplant procedure with no repercussions on the length of hospital stay, graft short- and long-term function and patient survival. However the procedure eads to a longer operative time and greater need for fluids and blood products.
\end{abstract}

\begin{abstract}
$\mathrm{A}$ UTOSOMAL-DOMINANT polycystic kidney disease (ADPKD) is a common cause of end-stage renal disease. Pretransplant native nephrectomy is performed less frequently today than some years ago. However, some patients have massively enlarged kidneys that make the transplant surgery difficult or impossible. Our study sought to evaluate whether patients with ADPKD can be safely and effectively transplanted when a native nephrectomy is performed simultaneously.
\end{abstract}

\section{PATIENTS AND METHODS}

From 1980 to 2005, we performed 1432 renal transplantations, including $159(11.1 \%)$ patients with end-stage renal disease owing to ADPKD. This retrospective study compared two groups of these 159 patients: 16 who needed a simultaneous nephrectomy during the transplant because of a space conflict between the massively enlarged ipsilateral native kidney and the graft $(\mathrm{N}+)$, and 143 who underwent the transplant without a need to remove a native kidney $(\mathrm{N} 0)$.

The statistical analysis was performed using SPSS for Windows 10.0. Categorical metrics were compared by $\chi^{2}$ testing, and continuous variables by Student's $t$ test, with Yates's correction or

From the Department of Urology and Renal Transplantation, Hospitais da Universidade de Coimbra, Coimbra, Portugal.

Address reprint requests to Pedro Nunes, Department of Urology and Renal Transplantation, Hospitais da Universidade de Coimbra, 3000 Coimbra, Portugal. E-mail: ptnunes@gmail.com

0041-1345/07/\$-see front matter doi:10.1016/j.transproceed.2007.07.035 
Fisher's exact test where appropriate. Graft and patient cumulative actuarial survival and survival curves were calculated by KaplanMeier analysis and tested for differences with the Mantel-Cox log-rank test. $P<.05$ was considered significant.

\section{RESULTS}

There were no significant differences regarding mean donor age $(\mathrm{N} 0=34.99$ vs $\mathrm{N}+=35.13$ years $)$, percentage of cadaveric donors $(\mathrm{N} 0=97.8 \%$ vs $\mathrm{N}+=93.8 \%)$, mean recipient age $(\mathrm{N} 0=50.10 \mathrm{vs} \mathrm{N}+=49.38$ years $)$, recipient gender $(\mathrm{N} 0=53.4 \%$ vs $\mathrm{N}+=66.7 \%$ males $)$, length of pretransplant dialysis ( $\mathrm{N} 0=32.30$ vs $\mathrm{N}+=29.44$ months $)$, or HLA matches $(\mathrm{N} 0=2.33$ vs $\mathrm{N}+=2.33)$. Surgical procedures were basically the same over time. Native nephrectomy was performed through a cranial extension of the original Gibson incision used for the transplant. Initial immunosuppressive schemes showed no significant differences between the groups.

A greater percentage of surgeries requiring $>3$ hours occurred in the $\mathrm{N}+$ group $(\mathrm{N} 0=38.6 \%$ vs $\mathrm{N}+=64.3 \%$; $P=.05)$, reflecting the longer surgery time in the group requiring nephrectomy (N0 3.01 vs $\mathrm{N}+=4.23$ hours; $P<$ $.001)$. This differences did not affect the mean cold ischemia time $(\mathrm{N} 0=20.57$ vs $\mathrm{N}+=19.90$ hours; $P=\mathrm{NS})$. The need for intraoperative cystalloid fluid (N0 1.84 vs N+ $=2.76 \mathrm{~L}$; $P<.001$ ), blood (N0 $=1.05$ vs. $\mathrm{N}+=1.81$ units; $P<.001)$ and fresh frozen plasma (N0 2.07 vs N+2.93 units, $P<.05$ ) transfusion were also greater for this group. Central venous pressure at the time of reperfusion was similar in both cohorts $\left(\mathrm{N} 0=11.85\right.$ vs $\left.\mathrm{N}+=12.15 \mathrm{~cm} \mathrm{H}_{2} \mathrm{O}\right)$.

Mean follow-up was 6.36 years for the N0 and 8.53 years for the $\mathrm{N}+$ groups $(P=\mathrm{NS})$. Surgical complications were no different $(\mathrm{N} 0=12.2 \%$ vs $\mathrm{N}+=6.3 \% ; P=\mathrm{NS})$. There was one urologic complication (ureteral stenosis) in the nephrectomy group $(6.3 \%)$ and $2.8 \%$ on the N0 group $(P=$ $.005)$. Delayed graft function $(\mathrm{N} 0=19.9 \%$ vs $\mathrm{N}+=$ $12.5 \%$ ), acute rejections ( $\mathrm{N} 0=25.5 \%$ vs $\mathrm{N}+=33.3 \%$ ), and chronic allograft dysfunction $(\mathrm{N} 0=15.8 \%$ vs $\mathrm{N}+=28.6 \%$ ) showed no significant differences.

Graft function assessed by serum creatinine was no different between the groups at 1 month $(\mathrm{N} 0=1.79 \mathrm{vs} \mathrm{N}+=1.60$ $\mathrm{mg} / \mathrm{dL}), 1$ year $(\mathrm{N} 0=1.38 \mathrm{vs} \mathrm{N}+=1.39 \mathrm{mg} / \mathrm{dL})$, or 5 years $(\mathrm{N} 0=1.29$ vs $\mathrm{N}+=1.47 \mathrm{mg} / \mathrm{dL})$.

Actuarial cumulative graft survivals at 1 and 5 years were $91.6 \%$ and $79.4 \%$ for group N0 and $93.3 \%$ and $86.4 \%$ for group $\mathrm{N}+$ respectively. Causes of graft loss did not differ between the two groups. Actuarial cumulative patient survivals at 1 and 5 years were $93.8 \%$ and $83.6 \%$ for group N0 and $93.3 \%$ and $93.3 \%$ for group $\mathrm{N}+$. Causes of death were similar.

\section{DISCUSSION}

The percentage of ADPKD in our series was comparable to other reports. ${ }^{1}$ In the past, many patients required unilateral or bilateral nephrectomy for absolute indications: pain, infection, hemorrhage, hypertension, and digestive symp- toms. With progresses in the conservative management of these situations-pain killers, antihypertensive medications, antibiotic therapy, and blood replacement-fewer kidneys have needed to be removed, so pretransplant indications for nephrectomy in patients with ADPKD are less frequent now than 20 years ago. There are some advantages in maintaining native kidneys in this disease, such as avoiding fluid overload, congestive heart failure, hyperkalemia, anemia, and renal osteodystrophy. ${ }^{2}$ Brazda et $\mathrm{al}^{3}$ observed higher graft and patient survival rates among ADPKD patients who had undergone a pretransplant unilateral nephrectomy than those without nephrectomy. Other workers have suggested benefits of concomitant bilateral nephrectomy and transplantation, with a reduction of symptoms and more space for the graft. ${ }^{4}$

The majority of patients with ADPKD now come to the transplant with their kidneys in situ. In the great majority of these patients, the kidney volume does not pose a problem for the transplant procedure. There are, however, some patients with massively enlarged kidneys, where safe grafting is precluded.

There are no imaging studies or clinical clues that can absolutely ensure the feasibility of a transplant procedure without the need to simultaneously perform a native nephrectomy. Sometimes, clinically or radiologically enormous organs are easily displaced or some cysts ruptured to gain room in the iliac fossa for the graft. Conversely, some apparently not so large kidneys are difficult to manage conservatively during the transplant, making nephrectomy obligatory.

Our study, like others, ${ }^{5,6}$ demonstrated that when a massively enlarged native kidney is encountered during the transplant procedure, it can be safely excised without compromising the graft or patient outcome. In all cases, we were able to perform the nephrectomy through an upper extension of the same incision originally planned for the grafting procedure. Future possibilities can be a laparoscopic approach at the same operation. ${ }^{7}$ The operative time increased a mean of 70 minutes, the need for fluids and blood products was greater, but the hemodynamic parameters were comparable at the moment of reperfusion, allowing similar short- and long-term outcomes for grafts and patients. There was only one surgical (urologic) complication in the nephrectomy group, allowing us to conclude that this is a safe procedure.

In our experience, there is no need to remove a massively enlarged ADPKD kidney before transplantation on the basis of lack of room for the graft. If needed, the nephrectomy can be completed at the time of the transplant with no increased risks for the graft and the patient.

\section{REFERENCES}

1. Gabow PA: Autosomal dominant polycystic kidney disease. N Engl J Med 329:332, 1993

2. Rozansk J, Kozlowska I, Myslak M, et al: Pretransplant nephrectomy in patients with autosomal dominant polycystic kidney disease. Transplant Proc 37:666, 2005 
3. Brazda E, Ofner D, Riedmann B, et al: The effect of nephrectomy on the outcome of renal transplantation in patients with polycystic kidney disease. Ann Transplant 1:15, 1996

4. Glassman DT, Nipkow L, Bartlett ST, et al: Bilateral nephrectomy with concomitant renal graft transplantation for autosomal dominant polycystic kidney disease. J Urol 164:661, 2000

5. Tabibi A, Simforoosh N, Abadpour P, et al: Concomitant nephrectomy of massively enlarged kidneys and renal transplanta- tion in autosomal dominant polycystic kidney disease. Transplant Proc 37:2939, 2005

6. Fuller TF, Brennan TV, Feng S, et al: End stage polycystic kidney disease: indications and timing of native nephrectomy relative to kidney transplantation. J Urol 174:2284, 2005

7. Gill IS, Kaouk JH, Hobart MG, et al: Laparoscopic bilateral synchronous nephrectomy for autosomal dominant polycystic kidney disease: the initial experience. J Urol 165:1093, 2001 